\title{
Global attractivity of a discrete Lotka-Volterra competition system with infinite delays and feedback controls
}

Daiyong $\mathrm{Wu}^{*}$

\section{"Correspondence:} wudy9901@163.com Department of Mathematics, Anqing Normal College, Anqing, Anhui 246133, China

\begin{abstract}
In this paper, we propose a discrete Lotka-Volterra competition system with infinite delays and feedback controls. Sufficient conditions which ensure the global attractivity of the system are obtained. An example together with its numerical simulation shows the feasibility of the main results.
\end{abstract}

Keywords: competition system; feedback control; discrete; global attractivity

\section{Introduction}

For the last decades, the ecological competition systems governed by differential equations of Lotka-Volterra type have been investigated extensively. Many interesting results concerned with the global existence and attractivity of periodic solution, persistence and extinction of the population, etc. have been obtained; we refer to [1-4] and the references therein. Already, many authors [5-21] have argued that the discrete time models governed by difference equations are more appropriate than the continuous ones when the populations have nonoverlapping generations. Particularly, the persistence, permanence, extinction, local and global stability and the existence of positive periodic solutions, etc., for discrete competitive systems are studied in [5, 9, 12, 13, 15-18, 21]. Chen and Zhou [9] discussed the following discrete Lotka-Volterra competition system:

$$
\left\{\begin{array}{l}
x_{1}(n+1)=x_{1}(n) \exp \left[r_{1}(n)\left(1-\frac{x_{1}(n)}{K_{1}(n)}-\mu_{1}(n) x_{2}(n)\right)\right], \\
x_{2}(n+1)=x_{2}(n) \exp \left[r_{2}(n)\left(1-\frac{x_{2}(n)}{K_{2}(n)}-\mu_{2}(n) x_{1}(n)\right)\right] .
\end{array}\right.
$$

They obtained sufficient conditions which guarantee the persistence of system (1.1). Also, for the periodic case, they obtained sufficient conditions for the existence of a globally stable periodic solution.

Chen [12] studied the following nonautonomous two-species discrete competitive systems with deviating arguments:

$$
\left\{\begin{array}{l}
x_{1}(n+1)=x_{1}(n) \exp \left[r_{1}(n)\left(1-\frac{x_{1}(n)}{K_{1}(n)}-\mu_{1}(n) \sum_{s=-\infty}^{n} H_{1}(n-s) x_{2}(s)\right)\right], \\
x_{2}(n+1)=x_{2}(n) \exp \left[r_{2}(n)\left(1-\frac{x_{2}(n)}{K_{2}(n)}-\mu_{2}(n) \sum_{s=-\infty}^{n} H_{2}(n-s) x_{1}(s)\right)\right] .
\end{array}\right.
$$

They obtained sufficient conditions for the permanence of system (1.2).

\section{Springer}

(c) 2013 Wu; licensee Springer. This is an Open Access article distributed under the terms of the Creative Commons Attribution License (http://creativecommons.org/licenses/by/2.0), which permits unrestricted use, distribution, and reproduction in any medium, provided the original work is properly cited. 
On the other hand, feedback control is the basic mechanism by which systems, whether mechanical, electrical or biological, maintain their equilibrium or homeostasis. In the higher life forms, the conditions under which life can continue are quite narrow. A change in body temperature of half a degree is generally a sign of illness. The homeostasis of the body is maintained through the use of feedback control [22]. A primary contribution of C.R. Darwin during the last century was the theory that feedback over long time periods is responsible for the evolution of species. In 1931 Volterra [23] explained the balance between two populations of fish in a closed pond using the theory of feedback. Later, a series of mathematical models have been established to describe the dynamics of feedback control systems; see [14, 16-20, 23-25] and the references therein.

The purpose of this paper is to study the global attractivity of the following discrete Lotka-Volterra competition system with infinite delays and feedback controls:

$$
\left\{\begin{aligned}
x_{1}(n+1)= & x_{1}(n) \exp \left[r_{1}-a_{11} x_{1}(n)-a_{12} \sum_{s=-\infty}^{n} H_{1}(n-s) x_{2}(s)\right. \\
& \left.-c_{1} \sum_{s=-\infty}^{n} H_{2}(n-s) \mu_{1}(s)\right], \\
x_{2}(n+1)= & x_{2}(n) \exp \left[r_{2}-a_{22} x_{2}(n)-a_{21} \sum_{s=-\infty}^{n} H_{3}(n-s) x_{1}(s)\right. \\
& \left.-c_{2} \sum_{s=-\infty}^{n} H_{4}(n-s) \mu_{2}(s)\right] \\
\mu_{1}(n+1)= & \left(1-a_{1}\right) \mu_{1}(n)+b_{1} \sum_{s=-\infty}^{n} H_{5}(n-s) x_{1}(s) \\
\mu_{2}(n+1)= & \left(1-a_{2}\right) \mu_{2}(n)+b_{2} \sum_{s=-\infty}^{n} H_{6}(n-s) x_{2}(s)
\end{aligned}\right.
$$

where $0<a_{i}<1, r_{i}, b_{i}, c_{i}, a_{i j} \in(0, \infty), i=1,2, j=1,2, x_{i}(n)(i=1,2)$ are the density of the $i$ species at time $n$ and $\mu_{i}(n)(i=1,2)$ are the control variables at time $n . H_{i}(n)(i=1,2, \ldots, 6)$ are bounded nonnegative sequences such that $\sum_{n=0}^{\infty} H_{i}(n)=1$.

By the biological meaning, we focus our discussion on the positive solutions of (1.3). So, it is assumed that the initial conditions of (1.3) are of the form

$$
x_{i}(s)=\Phi_{i}(s) \geq 0, \quad \Phi_{i}(0)>0, \quad \mu_{i}(s)=\Psi_{i}(s) \geq 0, \quad \Psi_{i}(0)>0, i=1,2,
$$

where $s=\ldots,-n,-n+1, \ldots,-1,0$. One can easily show that the solutions of (1.3) with (1.4) remain positive for all $n \in Z_{+}$, where $Z_{+}=\{0,1,2, \ldots\}$.

Further, assume

$$
\left(r_{1} a_{21}-r_{2} d_{1}\right)\left(r_{2} a_{12}-r_{1} d_{2}\right)>0
$$

where $d_{1}=\frac{1}{a_{1}}\left(a_{1} a_{11}+b_{1} c_{1}\right), d_{2}=\frac{1}{a_{2}}\left(a_{2} a_{22}+b_{2} c_{2}\right)$. Then system (1.3) has a unique positive equilibrium $\left(x_{1}^{*}, x_{2}^{*}, \mu_{1}^{*}, \mu_{2}^{*}\right)$ with

$$
x_{1}^{*}=\frac{r_{2} a_{12}-r_{1} d_{2}}{a_{12} a_{21}-d_{1} d_{2}}, \quad x_{2}^{*}=\frac{r_{1} a_{21}-r_{2} d_{1}}{a_{12} a_{21}-d_{1} d_{2}}, \quad \mu_{1}^{*}=\frac{a_{1}}{b_{1}} x_{1}^{*}, \quad \mu_{2}^{*}=\frac{a_{2}}{b_{2}} x_{2}^{*} .
$$

The aim of this paper is, by developing the analysis technique of Chen [12], Liao and $\mathrm{Yu}$ [14], Chen and Teng [15], to obtain a set of sufficient conditions for the global attractivity of system (1.3). The paper is organized as follows. In Section 2, as preliminaries, some useful lemmas are given. In Section 3, we study the global attractivity of positive equilibrium of system (1.3). In Section 4, the numerical simulations on the global attractivity of equilibrium are given. 


\section{Preliminaries}

In this section, we introduce some auxiliary lemmas which will be useful in the following.

Lemma 1 (see [15]) Let the function $f(u)=u \exp (\alpha-\beta u)$, where $\alpha$ and $\beta$ are positive constants. Then $f(u)$ is nondecreasing on $u \in\left(0, \frac{1}{\beta}\right]$.

Lemma 2 (see [15]) Assume that the sequence $\{u(n)\}$ satisfies

$$
u(n+1)=u(n) \exp (\alpha-\beta u(n)), \quad n=1,2, \ldots,
$$

where $\alpha$ and $\beta$ are positive constants and $u(0)>0$. We have

(i) if $\alpha<2$, then $\lim _{n \rightarrow \infty} u(n)=\frac{\alpha}{\beta}$.

(ii) if $\alpha \leq 1$, then $u(n) \leq \frac{1}{\beta}$ for all $n=2,3, \ldots$.

Lemma 3 (see [8]) Suppose that functions $f, g: Z_{+} \times[0, \infty) \rightarrow[0, \infty)$ satisfy $f(n, x) \leq$ $g(n, x)(f(n, x) \geq g(n, x))$ for $n \in Z_{+}$and $x \in[0, \infty)$ and $g(n, x)$ is nondecreasing with respect to $x>0$. If sequences $\{x(n)\}$ and $\{u(n)\}$ are the nonnegative solutions of the following difference equations:

$$
x(n+1)=f(n, x(n)), \quad u(n+1)=g(n, u(n)), \quad n=0,1,2, \ldots,
$$

respectively, and $x(0) \leq u(0)(x(0) \geq u(0))$, then for all $n \geq 0$, we have

$$
x(n) \leq u(n)(x(n) \geq u(n))
$$

Lemma 4 (see [12]) Let $x: Z \rightarrow R$ be a nonnegative bounded sequence, and let $H$ : $Z_{+} \rightarrow R$ be a nonnegative sequence such that $\sum_{n=0}^{\infty} H(n)=1$, where $Z=\{0, \pm 1, \pm 2, \ldots\}$, $R=(-\infty, \infty)$. Then

$$
\liminf _{n \rightarrow+\infty} x(n) \leq \liminf _{n \rightarrow+\infty} \sum_{s=-\infty}^{n} H(n-s) x(s) \leq \limsup _{n \rightarrow+\infty} \sum_{s=-\infty}^{n} H(n-s) x(s) \leq \limsup _{n \rightarrow+\infty} x(n) .
$$

We further consider the following discrete linear equation:

$$
u(n+1)=\left(1-\gamma_{1}\right) u(n)+\gamma_{2} \sum_{s=-\infty}^{n} H(n-s) x(s), \quad n \in Z_{+}
$$

where $0<\gamma_{1}<1, \gamma_{2} \in(0, \infty)$. $H(n)$ is a nonnegative sequence defined on $Z_{+}$such that $\sum_{n=0}^{\infty} H(n)=1$ and $x(n)$ is a nonnegative bounded sequence defined on $Z$ with

$$
x_{*} \leq \liminf _{n \rightarrow \infty} x(n) \leq \limsup _{n \rightarrow \infty} x(n) \leq x^{*},
$$

where $x_{*}, x^{*}$ are nonnegative constants.

Lemma 5 Any solution of system (2.1) with $u(0)>0$ satisfies

$$
\frac{\gamma_{2}}{\gamma_{1}} x_{*} \leq \liminf _{n \rightarrow \infty} u(n) \leq \limsup _{n \rightarrow \infty} u(n) \leq \frac{\gamma_{2}}{\gamma_{1}} x^{*}
$$


Proof From Lemma 4,

$$
\limsup _{n \rightarrow+\infty} \sum_{s=-\infty}^{n} H(n-s) x(s) \leq \limsup _{n \rightarrow+\infty} x(n) \leq x^{*} .
$$

Hence, for each $\varepsilon>0$, there exists an enough large integer $n_{0}$ such that for $n \geq n_{0}$,

$$
\sum_{s=-\infty}^{n} H(n-s) x(n) \leq x^{*}+\varepsilon
$$

By system (2.1), we can obtain

$$
\begin{aligned}
u(n) & =u\left(n_{0}\right)\left(1-\gamma_{1}\right)^{n-n_{0}}+\gamma_{2} \sum_{i=n_{0}}^{n-1}\left[\left(1-\gamma_{1}\right)^{n-i-1} \sum_{s=-\infty}^{i} H(i-s) x(s)\right] \\
& \leq u_{0}\left(1-\gamma_{1}\right)^{n-n_{0}}+\gamma_{2}\left(x^{*}+\varepsilon\right) \sum_{i=n_{0}}^{n-1}\left(1-\gamma_{1}\right)^{n-i-1} \\
& =u_{0}\left(1-\gamma_{1}\right)^{n-n_{0}}+\gamma_{2}\left(x^{*}+\varepsilon\right) \frac{1-\left(1-\gamma_{1}\right)^{n-n_{0}}}{\gamma_{1}} \rightarrow \frac{\gamma_{2}\left(x^{*}+\varepsilon\right)}{\gamma_{1}}, \quad n \rightarrow \infty .
\end{aligned}
$$

Thus,

$$
\limsup _{n \rightarrow \infty} u(n) \leq \frac{\gamma_{2}\left(x^{*}+\varepsilon\right)}{\gamma_{1}} .
$$

By the arbitrariness of $\varepsilon$, we can obtain

$$
\limsup _{n \rightarrow \infty} u(n) \leq \frac{\gamma_{2}}{\gamma_{1}} x^{*}
$$

We can prove $\liminf _{n \rightarrow \infty} u(n) \geq \frac{\gamma_{2}}{\gamma_{1}} x_{*}$ in a similar way. Thus, we complete the proof.

Lemma 6 Assume $\lim _{n \rightarrow \infty} x(n)=\bar{x}$. For every solution $u(n)$ of equation (2.1), we have

$$
\lim _{n \rightarrow \infty} u(n)=\frac{\gamma_{2}}{\gamma_{1}} \bar{x} .
$$

By Lemma 5, the proof of Lemma 6 is obtained easily. Hence, we omit it here.

\section{Global attractivity}

In this section, we derive sufficient conditions which guarantee that the positive equilibrium of system (1.3) is globally attractive. The technique of proofs is to use an iteration scheme.

Theorem 1 Assume

$$
\left(r_{1} a_{21}-r_{2} d_{1}\right)\left(r_{2} a_{12}-r_{1} d_{2}\right)>0
$$

and

$$
\frac{r_{2} a_{12}}{a_{22}}+\frac{b_{1} c_{1} r_{1}}{a_{1} a_{11}}<r_{1} \leq 1, \quad \frac{r_{1} a_{21}}{a_{11}}+\frac{b_{2} c_{2} r_{2}}{a_{2} a_{22}}<r_{2} \leq 1 .
$$

Then equilibrium $\left(x_{1}^{*}, x_{2}^{*}, \mu_{1}^{*}, \mu_{2}^{*}\right)$ of system (1.3) with (1.4) is globally attractive. 
Proof Let $\left(x_{1}(n), x_{2}(n), \mu_{1}(n), \mu_{2}(n)\right)$ be any solution of system (1.3) with (1.4). Denote

$$
U_{i}=\limsup _{n \rightarrow \infty} x_{i}(n), \quad V_{i}=\liminf _{n \rightarrow \infty} x_{i}(n), \quad i=1,2,
$$

and

$$
P_{i}=\limsup _{n \rightarrow \infty} \mu_{i}(n), \quad Q_{i}=\liminf _{n \rightarrow \infty} \mu_{i}(n), \quad i=1,2 .
$$

We now claim that $U_{i}=V_{i}=x_{i}^{*}, P_{i}=Q_{i}=\mu_{i}^{*}, i=1,2$.

From the first equation of system (1.3), we obtain

$$
x_{1}(n+1) \leq x_{1}(n) \exp \left[r_{1}-a_{11} x_{1}(n)\right], \quad n=0,1,2, \ldots
$$

Consider the auxiliary equation

$$
p(n+1)=p(n) \exp \left[r_{1}-a_{11} p(n)\right]
$$

From $r_{1} \leq 1$, by the conclusion (ii) of Lemma 2, we have that $p(n) \leq \frac{1}{a_{11}}$ for all $n \geq 2$, where $p(n)$ is any solution of equation (3.1) with initial value $p(0)>0$. From Lemma 1 , we have $f(p)=p \exp \left(r_{1}-a_{11} p\right)$ is nondecreasing for $p \in\left(0, \frac{1}{a_{11}}\right]$.

Hence, from Lemma 3 , we obtain $x_{1}(n) \leq p(n)$ for all $n \geq 2$, where $p(n)$ is the solution of equation (3.1) with $p(2)=x_{1}(2)$. Further, combining it with the conclusion (i) of Lemma 2, we obtain

$$
U_{1}=\limsup _{n \rightarrow \infty} x_{1}(n) \leq \lim _{n \rightarrow \infty} p(n)=\frac{r_{1}}{a_{11}}:=M_{1}^{1} .
$$

From the second equation of system (1.3), we obtain

$$
x_{2}(n+1) \leq x_{2}(n) \exp \left[r_{2}-a_{22} x_{2}(n)\right], \quad n=0,1,2, \ldots
$$

By a similar argument as that above, we have

$$
U_{2}=\limsup _{n \rightarrow \infty} x_{2}(n) \leq \frac{r_{2}}{a_{22}}:=M_{1}^{2} .
$$

By Lemma 4 and Lemma 5, we obtain

$$
P_{i}=\limsup _{n \rightarrow \infty} \mu_{i}(n) \leq \frac{b_{i}}{a_{i}} M_{1}^{i}, \quad i=1,2 .
$$

Then, for any constant $\varepsilon>0$ sufficiently small, there is an integer $n_{1}>2$ such that if $n \geq n_{1}$, then

$$
x_{i}(n) \leq M_{1}^{i}+\varepsilon, \quad \mu_{i}(n) \leq \frac{b_{i}}{a_{i}} M_{1}^{i}+\varepsilon, \quad i=1,2 .
$$

Further, from Lemma 4 and the first equation of system (1.3), we have

$$
x_{1}(n+1) \geq x_{1}(n) \exp \left[r_{1}-a_{11} x_{1}(n)-a_{12}\left(M_{1}^{2}+\varepsilon\right)-c_{1}\left(\frac{b_{1}}{a_{1}} M_{1}^{1}+\varepsilon\right)\right], \quad n \geq n_{1}
$$


Consider the auxiliary equation

$$
p(n+1)=p(n) \exp \left[r_{1}-a_{11} p(n)-a_{12}\left(M_{1}^{2}+\varepsilon\right)-c_{1}\left(\frac{b_{1}}{a_{1}} M_{1}^{1}+\varepsilon\right)\right], \quad n \geq n_{1} \text {. }
$$

From $\frac{r_{2} a_{12}}{a_{22}}+\frac{b_{1} c_{1} r_{1}}{a_{1} a_{11}}<r_{1} \leq 1$ and the arbitrariness of $\varepsilon>0$, we have

$$
0<r_{1}-a_{12}\left(M_{1}^{2}+\varepsilon\right)-c_{1}\left(\frac{b_{1}}{a_{1}} M_{1}^{1}+\varepsilon\right)<1
$$

By the conclusion (ii) of Lemma 2, we have that $p(n) \leq \frac{1}{a_{11}}$ for all $n \geq n_{1}$, where $p(n)$ is any solution of equation (3.2) with initial value $p\left(n_{1}\right)>0$. From Lemma 1 , we have

$$
f(p)=\exp \left[r_{1}-a_{11} p-a_{12}\left(M_{1}^{2}+\varepsilon\right)-c_{1}\left(\frac{b_{1}}{a_{1}} M_{1}^{1}+\varepsilon\right)\right]
$$

is nondecreasing for $p \in\left(0, \frac{1}{a_{11}}\right]$.

Hence, from Lemma 3, we have $x_{1}(n) \geq p(n)$ for all $n \geq n_{1}$, where $p(n)$ is the solution of equation (3.2) with $p\left(n_{1}\right)=x_{1}\left(n_{1}\right)$. Combining it with the conclusion (i) of Lemma 2 , we obtain

$$
V_{1}=\liminf _{n \rightarrow \infty} x_{1}(n) \geq \lim _{n \rightarrow \infty} p(n)=\frac{1}{a_{11}}\left[r_{1}-a_{12}\left(M_{1}^{2}+\varepsilon\right)-c_{1}\left(\frac{b_{1}}{a_{1}} M_{1}^{1}+\varepsilon\right)\right] .
$$

From the arbitrariness of $\varepsilon>0$, we conclude $V_{1} \geq m_{1}^{1}$, where

$$
m_{1}^{1}=\frac{1}{a_{11}}\left[r_{1}-a_{12} M_{1}^{2}-c_{1} \frac{b_{1}}{a_{1}} M_{1}^{1}\right]
$$

From Lemma 4 and the second equation of system (1.3), we further have

$$
x_{2}(n+1) \geq x_{2}(n) \exp \left[r_{2}-a_{22} x_{2}(n)-a_{21}\left(M_{1}^{1}+\varepsilon\right)-c_{2}\left(\frac{b_{2}}{a_{2}} M_{1}^{2}+\varepsilon\right)\right], \quad n \geq n_{1} \text {. }
$$

By a similar argument as that above, we can obtain

$$
V_{2}=\liminf _{n \rightarrow \infty} x_{2}(n) \geq \frac{1}{a_{22}}\left[r_{2}-a_{21} M_{1}^{2}-c_{1} \frac{b_{1}}{a_{1}} M_{1}^{1}\right]:=m_{1}^{2} \text {. }
$$

By Lemma 4 and Lemma 5, we further obtain

$$
Q_{i}=\liminf _{n \rightarrow \infty} \mu_{i}(n) \geq \frac{b_{i}}{a_{i}} m_{1}^{i}, \quad i=1,2
$$

Hence, for $\varepsilon>0$ sufficiently small, there is an $n_{2}>n_{1}$ such that if $n \geq n_{2}$, then

$$
x_{i}(n) \geq m_{1}^{i}-\varepsilon, \quad \mu_{i}(n) \geq \frac{b_{i}}{a_{i}} m_{1}^{i}-\varepsilon, \quad i=1,2 .
$$

From Lemma 4 and the first equation of system (1.3), we further have

$$
x_{1}(n+1) \leq x_{1}(n) \exp \left[r_{1}-a_{11} x_{1}(n)-a_{12}\left(m_{1}^{2}-\varepsilon\right)-c_{1}\left(\frac{b_{1}}{a_{1}} m_{1}^{1}-\varepsilon\right)\right], \quad n \geq n_{2} .
$$


Consider the auxiliary equation

$$
p(n+1) \leq p(n) \exp \left[r_{1}-a_{11} p(n)-a_{12}\left(m_{1}^{2}-\varepsilon\right)-c_{1}\left(\frac{b_{1}}{a_{1}} m_{1}^{1}-\varepsilon\right)\right], \quad n \geq n_{2} .
$$

From $\frac{r_{2} a_{12}}{a_{22}}+\frac{b_{1} c_{1} r_{1}}{a_{1} a_{11}}<r_{1} \leq 1$ and the arbitrariness of $\varepsilon>0$, we have

$$
0<r_{1}-a_{12}\left(m_{1}^{2}-\varepsilon\right)-c_{1}\left(\frac{b_{1}}{a_{1}} m_{1}^{1}-\varepsilon\right)<1 .
$$

Similarly to the above discussion, we can obtain

$$
U_{1}=\liminf _{n \rightarrow \infty} x_{1}(n) \leq \lim _{n \rightarrow \infty} p(n)=\frac{1}{a_{11}}\left[r_{1}-a_{12}\left(m_{1}^{2}-\varepsilon\right)-c_{1}\left(\frac{b_{1}}{a_{1}} m_{1}^{1}-\varepsilon\right)\right] .
$$

From the arbitrariness of $\varepsilon>0$, we conclude $U_{1} \leq M_{2}^{1}$, where

$$
M_{2}^{1}=\frac{1}{a_{11}}\left[r_{1}-a_{12} m_{1}^{2}-c_{1} \frac{b_{1}}{a_{1}} m_{1}^{1}\right] .
$$

From Lemma 4 and the second equation of system (1.3), we further have

$$
x_{2}(n+1) \leq x_{2}(n) \exp \left[r_{2}-a_{22} x_{2}(n)-a_{21}\left(m_{1}^{1}-\varepsilon\right)-c_{2}\left(\frac{b_{2}}{a_{2}} m_{1}^{2}-\varepsilon\right)\right], \quad n \geq n_{2} .
$$

By a similar argument as that above, we can obtain

$$
U_{2}=\liminf _{n \rightarrow \infty} x_{2}(n) \leq \frac{1}{a_{22}}\left[r_{2}-a_{21} m_{1}^{1}-c_{2} \frac{b_{2}}{a_{2}} m_{1}^{2}\right]:=M_{2}^{2} .
$$

By Lemma 4 and Lemma 5 , we obtain

$$
P_{i}=\limsup _{n \rightarrow \infty} \mu_{i}(n) \leq \frac{b_{i}}{a_{i}} M_{2}^{i}, \quad i=1,2 .
$$

Hence, for $\varepsilon>0$ sufficiently small, there is an $n_{3}$ such that if $n \geq n_{3}$,

$$
x_{i}(n) \leq M_{2}^{i}+\varepsilon, \quad \mu_{i}(n) \leq \frac{b_{i}}{a_{i}} M_{2}^{i}+\varepsilon, \quad i=1,2 .
$$

From Lemma 4 and the first equation of system (1.3), we have

$$
x_{1}(n+1) \geq x_{1}(n) \exp \left[r_{1}-a_{11} x_{1}(n)-a_{12}\left(M_{2}^{2}+\varepsilon\right)-c_{1}\left(\frac{b_{1}}{a_{1}} M_{2}^{1}+\varepsilon\right)\right], \quad n \geq n_{3} .
$$

Consider the auxiliary equation

$$
p(n+1)=p(n) \exp \left[r_{1}-a_{11} p(n)-a_{12}\left(M_{2}^{2}+\varepsilon\right)-c_{1}\left(\frac{b_{1}}{a_{1}} M_{2}^{1}+\varepsilon\right)\right], \quad n \geq n_{3} .
$$

Since

$$
0<r_{1}-a_{12}\left(M_{2}^{2}+\varepsilon\right)-c_{1}\left(\frac{b_{1}}{a_{1}} M_{2}^{1}+\varepsilon\right)<1,
$$


similarly to the above discussion, we obtain

$$
V_{1}=\liminf _{n \rightarrow \infty} x_{1}(n) \geq \lim _{n \rightarrow \infty} p(n)=\frac{1}{a_{11}}\left[r_{1}-a_{12}\left(M_{2}^{2}+\varepsilon\right)-c_{1}\left(\frac{b_{1}}{a_{1}} M_{2}^{1}+\varepsilon\right)\right] .
$$

From the arbitrariness of $\varepsilon>0$, we conclude $V_{1} \geq m_{2}^{1}$, where

$$
m_{2}^{1}=\frac{1}{a_{11}}\left[r_{1}-a_{12} M_{2}^{2}-c_{1} \frac{b_{1}}{a_{1}} M_{2}^{1}\right]
$$

From Lemma 4 and the second equation of system (1.3), we further have

$$
x_{2}(n+1) \geq x_{2}(n) \exp \left[r_{2}-a_{22} x_{2}(n)-a_{21}\left(M_{2}^{1}+\varepsilon\right)-c_{2}\left(\frac{b_{2}}{a_{2}} M_{2}^{2}+\varepsilon\right)\right], \quad n \geq n_{3} .
$$

By a similar argument as that above, we can obtain

$$
V_{2}=\liminf _{n \rightarrow \infty} x_{2}(n) \geq \frac{1}{a_{22}}\left[r_{2}-a_{21} M_{2}^{1}-c_{2} \frac{b_{2}}{a_{2}} M_{2}^{2}\right]:=m_{2}^{2} .
$$

Continuing the above process, we can obtain four sequences $\left\{M_{n}^{i}\right\},\left\{m_{n}^{i}\right\}, i=1,2$ such that

$$
M_{n+1}^{1}=\frac{1}{a_{11}}\left[r_{1}-a_{12} m_{n}^{2}-c_{1} \frac{b_{1}}{a_{1}} m_{n}^{1}\right], \quad M_{n+1}^{2}=\frac{1}{a_{22}}\left[r_{2}-a_{21} m_{n}^{1}-c_{2} \frac{b_{2}}{a_{2}} m_{n}^{2}\right]
$$

and

$$
m_{n}^{1}=\frac{1}{a_{11}}\left[r_{1}-a_{12} M_{n}^{2}-c_{1} \frac{b_{1}}{a_{1}} M_{n}^{1}\right], \quad m_{n}^{2}=\frac{1}{a_{22}}\left[r_{2}-a_{21} M_{n}^{1}-c_{2} \frac{b_{2}}{a_{2}} M_{n}^{2}\right] .
$$

Clearly, we have

$$
m_{n}^{i} \leq V_{i} \leq U_{i} \leq M_{n}^{i}, \quad i=1,2
$$

Now, by means of the inductive method, we prove $\left\{M_{n}^{i}\right\}$ is monotonically decreasing, $\left\{m_{n}^{i}\right\}$ is monotonically increasing, $i=1,2$.

Firstly, it is clear that $M_{2}^{i} \leq M_{1}^{i}, m_{2}^{i} \geq m_{1}^{i}, i=1,2$. For $n=k(k \geq 2)$, we assume $M_{k}^{i} \leq M_{k-1}^{i}$ and $m_{k}^{i} \geq m_{k-1}^{i}, i=1,2$, then we have

$$
\begin{aligned}
& M_{k+1}^{1}=\frac{1}{a_{11}}\left[r_{1}-a_{12} m_{k}^{2}-c_{1} \frac{b_{1}}{a_{1}} m_{k}^{1}\right] \leq \frac{1}{a_{11}}\left[r_{1}-a_{12} m_{k-1}^{2}-c_{1} \frac{b_{1}}{a_{1}} m_{k-1}^{1}\right]=M_{k}^{1}, \\
& M_{k+1}^{2}=\frac{1}{a_{22}}\left[r_{2}-a_{21} m_{k}^{1}-c_{2} \frac{b_{2}}{a_{2}} m_{k}^{2}\right] \leq \frac{1}{a_{22}}\left[r_{2}-a_{21} m_{k-1}^{1}-c_{2} \frac{b_{2}}{a_{2}} m_{k-1}^{2}\right]=M_{k}^{2}
\end{aligned}
$$

and

$$
\begin{aligned}
& m_{k+1}^{1}=\frac{1}{a_{11}}\left[r_{1}-a_{12} M_{k+1}^{2}-c_{1} \frac{b_{1}}{a_{1}} M_{k+1}^{1}\right] \geq \frac{1}{a_{11}}\left[r_{1}-a_{12} M_{k}^{2}-c_{1} \frac{b_{1}}{a_{1}} M_{k}^{1}\right]=m_{k}^{1}, \\
& m_{k+1}^{2}=\frac{1}{a_{22}}\left[r_{2}-a_{21} M_{k+1}^{1}-c_{2} \frac{b_{2}}{a_{2}} M_{k+1}^{2}\right] \geq \frac{1}{a_{22}}\left[r_{2}-a_{21} M_{k}^{1}-c_{2} \frac{b_{2}}{a_{2}} M_{k}^{2}\right]=m_{k}^{2} .
\end{aligned}
$$




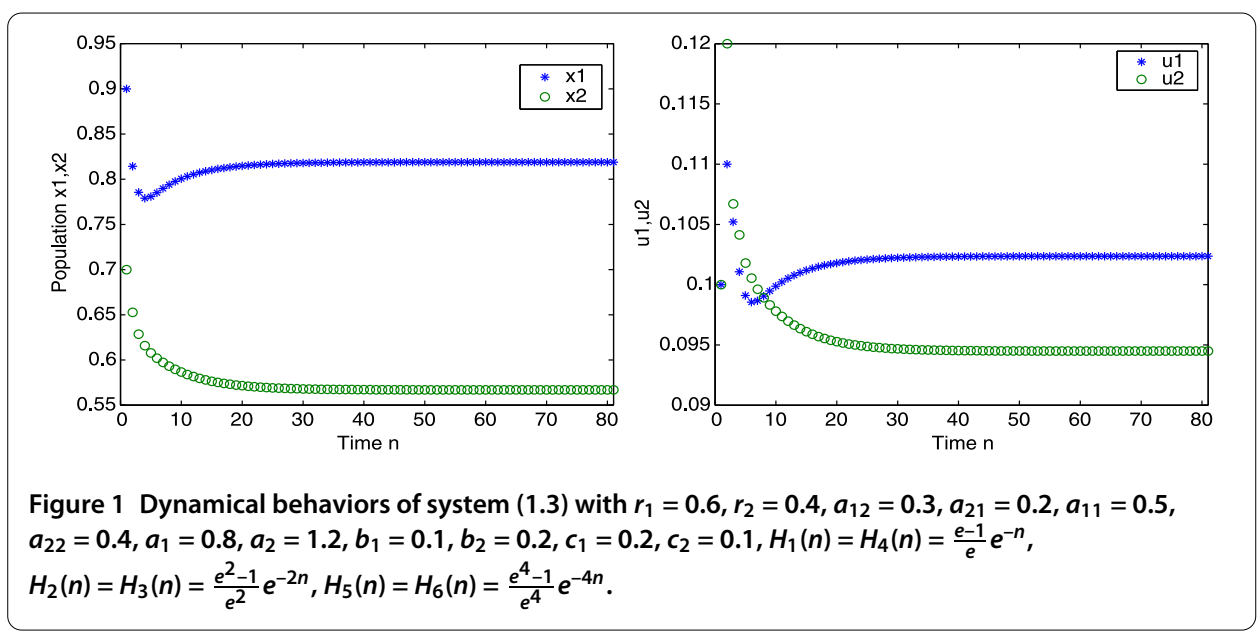

Therefore, $\left\{M_{n}^{i}\right\}$ is monotonically decreasing, $\left\{m_{n}^{i}\right\}$ is monotonically increasing, $i=1,2$. Consequently, $\lim _{n \rightarrow \infty} M_{n}^{i}$ and $\lim _{n \rightarrow \infty} m_{n}^{i}$ both exist, $i=1,2$. Let

$$
\lim _{n \rightarrow \infty} M_{n}^{i}=\bar{x}_{i}, \quad \lim _{n \rightarrow \infty} m_{n}^{i}=\bar{y}_{i}, \quad i=1,2
$$

From (3.3) and (3.4), we obtain

$$
\left\{\begin{array}{l}
a_{11} \bar{x}_{1}+a_{12} \bar{y}_{2}+\frac{b_{1} c_{1}}{a_{1}} \bar{y}_{1}=r_{1} \\
a_{22} \bar{x}_{2}+a_{21} \bar{y}_{1}+\frac{b_{2} c_{2}}{a_{2}} \bar{y}_{2}=r_{2} \\
a_{11} \bar{y}_{1}+a_{12} \bar{x}_{2}+\frac{b_{1} c_{1}}{a_{1}} \bar{x}_{1}=r_{1} \\
a_{22} \bar{y}_{2}+a_{21} \bar{x}_{1}+\frac{b_{2} c_{2}}{a_{2}} \bar{x}_{2}=r_{2}
\end{array}\right.
$$

It is clear that $\left(x_{1}^{*}, x_{2}^{*}, \mu_{1}^{*}, \mu_{2}^{*}\right)$ is a unique solution of equations (3.5). Therefore,

$$
U_{i}=V_{i}=\lim _{n \rightarrow \infty} x_{i}(n)=x_{i}^{*}, \quad i=1,2 .
$$

Further, by Lemma 6 , we can obtain $\lim _{n \rightarrow \infty} \mu_{i}(n)=\mu_{i}^{*}, i=1,2$. Thus, we complete the proof of Theorem 1 .

\section{Example}

The following example shows the feasibility of the main results.

Example 1 Choose $r_{1}=0.6, r_{2}=0.4, a_{12}=0.3, a_{21}=0.2, a_{11}=0.5, a_{22}=0.4, a_{1}=0.8, a_{2}=$ $1.2, b_{1}=0.1, b_{2}=0.2, c_{1}=0.2, c_{2}=0.1, H_{1}(n)=H_{4}(n)=\frac{e-1}{e} e^{-n}, H_{2}(n)=H_{3}(n)=\frac{e^{2}-1}{e^{2}} e^{-2 n}$, $H_{5}(n)=H_{6}(n)=\frac{e^{4}-1}{e^{4}} e^{-4 n}$ in system (1.3). By calculating, we have that positive equilibrium $(0.8189,0.5669,0.1024,0.0945),\left(r_{1} a_{21}-r_{2} d_{1}\right)\left(r_{2} a_{12}-r_{1} d_{2}\right)=0.0117, r_{1}-\left(\frac{r_{2} a_{12}}{a_{22}}+\frac{b_{1} c_{1} r_{1}}{a_{1} a_{11}}\right)=$ $0.27, r_{2}-\left(\frac{r_{1} a_{21}}{a_{11}}+\frac{b_{2} c_{2} r_{2}}{a_{2} a_{22}}\right)=0.1433$. Since

$$
\begin{aligned}
& \left(r_{1} a_{21}-r_{2} d_{1}\right)\left(r_{2} a_{12}-r_{1} d_{2}\right)>0, \quad\left(\frac{r_{2} a_{12}}{a_{22}}+\frac{b_{1} c_{1} r_{1}}{a_{1} a_{11}}\right)<r_{1} \leq 1, \\
& \left(\frac{r_{1} a_{21}}{a_{11}}+\frac{b_{2} c_{2} r_{2}}{a_{2} a_{22}}\right)<r_{2} \leq 1,
\end{aligned}
$$


the conditions of Theorem 1 hold. So, equilibrium $(0.8189,0.5669,0.1024,0.0945)$ is globally attractive.

Choose initial values $\left(x_{1}(s), x_{2}(s), \mu_{1}(s), \mu_{2}(s)\right)=(0.9,0.7,0.2,0.1), s=\ldots,-n,-n+1, \ldots$, $-1,0$.

By the numerical simulation (see Figure 1), we find that the solution $\left(x_{1}(n), x_{2}(n), \mu_{1}(n)\right.$, $\left.\mu_{2}(n)\right)$ turns to equilibrium $(0.8189,0.5669,0.1024,0.0945)$ as $n \rightarrow \infty$.

\section{Competing interests}

The author declares that he has no competing interests.

\section{Author's contributions}

The author carried out the proof of the theorem and approved the final manuscript.

\section{Acknowledgements}

The author would like to thank the main editor and anonymous referees for their valuable comments and suggestions leading to improvement of this paper. This work was supported by the Scientific Research Programmes of Colleges in Anhui (KJ2011A197)

Received: 20 July 2012 Accepted: 3 January 2013 Published: 18 January 2013

\section{References}

1. Ahmad, S: On the nonautonomous Lotka-Volterra competition equation. Proc. Am. Math. Soc. 117, $199-204$ (1993)

2. Ahmad, S, Lazer, AC: Average growth and extinction in a Lotka-Volterra system. Nonlinear Anal. 62, 545-557 (2005)

3. Ahmad, S, Lazer, AC: Average conditions for global asymptotic stability in a nonautonomous Lotka-Volterra system. Nonlinear Anal. 40, 37-49 (2000)

4. Tang, X, Zou, X: On positive periodic solutions of Lotka-Volterra competition systems with deviating arguments. Proc. Am. Math. Soc. 134, 2967-2974 (2006)

5. Liao, X, Zhou, S, Chen, Y: On permanence and global stability in a general Gilpin-Ayala competition predator-prey discrete system. Appl. Math. Comput. 190, 500-509 (2007)

6. Zhou, Z, Zou, X: Stable periodic solutions in a discrete periodic logistic equation. Appl. Math. Lett. 16(2), 165-171 (2003)

7. Liu, X: A note on the existence of periodic solution in discrete predator-prey models. Appl. Math. Model. 34, 2477-2483 (2010)

8. Wang, L, Wang, M: Ordinary Difference Equation. Xinjing Univ. Press, Xinjiang (1989)

9. Chen, YM, Zhou, Z: Stable periodic solution of a discrete periodic Lotka-Volterra competition system. J. Math. Appl. 277(1), 358-366 (2003)

10. Yang, XT: Uniform persistence and periodic solutions for a discrete predator-prey system with delays. J. Math. Anal. Appl. 316(1), 161-177 (2006)

11. Wang, K: Permanence and global asymptotical stability of a predator-prey model with mutual interference. Nonlinear Anal., Real World Appl. 12, 1062-1071 (2011)

12. Chen, FD: Permanence in a discrete Lotka-Volterra competition model with deviating arguments. Nonlinear Anal, Real World Appl. 9, 2150-2155 (2008)

13. Xiu, JB, Teng, ZD, Jiang, HJ: Permanence and global attractivity for discrete nonautonomous two-species Lotka-Volterra competitive system with delays and feedback controls. Period. Math. Hung. 63(1), 19-45 (2011)

14. Liao, LS, Yu, JS, Wang, L: Global attractivity in a logistic difference model with a feedback control. Comput. Math. Appl. 44, 1403-1411 (2002)

15. Chen, GY, Teng, ZD: On the stability in a discrete two-species competition system. J. Appl. Math. Comput. 38, 25-36 (2012)

16. Kong, XZ, Chen, LP, Yang, WS: Note on the persistent property of a discrete Lotka-Volterra competition system with delays and feedback controls. Adv. Differ. Equ. 2, 9 (2010)

17. Liao, X, Zhou, S, Chen, Y: Permanence and global stability in a discrete $n$-species competition system with feedback controls. Nonlinear Anal., Real World Appl. 9, 1661-1671 (2008)

18. Liao, X, Ouyang, Z, Zhou, S: Permanence of species in nonautonomous discrete Lotka-Volterra competition system with delays and feedback controls. J. Comput. Appl. Math. 211, 1-10 (2008)

19. Li, YK, Zhang, TW: Permanence and almost periodic sequence solution for a discrete delay logistic equation with feedback control. Nonlinear Anal., Real World Appl. 12, 1850-1864 (2011)

20. Li, QY, Liu, HW, Zhang, FQ: The permanence and extinction of a discrete predator-prey system with time delay and feedback controls. Adv. Differ. Equ. 2, 20 (2010)

21. Yu, ZX, Li, Z: Permanence and global attractivity of a discrete two-prey one-predator model with infinite delay. Discrete Dyn. Nat. Soc. (2009). doi:10.1155/2009/732510

22. Wiener, N: Cybernetics or Control and Communication in the Animal and the Machine. MIT Press, Cambridge (1948)

23. Gopalsamy, K: Stability and Oscillations in Delay Differential Equations of Population Dynamics. Kluwer Academic, Boston (1992)

24. Gopalsamy, K, Weng, PX: Feedback regulation of logistic growth. Int. J. Math. Math. Sci. 16, 177-192 (1993)

25. Liao, LS: Feedback regulation of a logistic growth variable coefficients. J. Math. Appl. 259(2), 489-500 (2001) 
doi:10.1186/1687-1847-2013-14

Cite this article as: Wu: Global attractivity of a discrete Lotka-Volterra competition system with infinite delays and feedback controls. Advances in Difference Equations 2013 2013:14.

Submit your manuscript to a SpringerOpen ${ }^{\circ}$ journal and benefit from:

- Convenient online submission

- Rigorous peer review

- Immediate publication on acceptance

- Open access: articles freely available online

- High visibility within the field

- Retaining the copyright to your article

Submit your next manuscript at $\gg$ springeropen.com 